\title{
SYSTEMATIC DECISION MAKING: A EXTENDED MULTI-CRITERIA DECISION MAKING MODEL
}

\author{
Xiaohan YUa, Zeshui XU'b , Junquan HUc, Shousheng LIU ${ }^{\mathrm{b}}$ \\ ${ }^{a}$ College of Command Information Systems, PLA University of Science and Technology, \\ 210007 Nanjing, China \\ ${ }^{b}$ College of Sciences, PLA University of Science and Technology, 211101 Nanjing, China \\ ${ }^{c}$ College of Communications Engineering, PLA University of Science and Technology, \\ 210007 Nanjing, China
}

Received 16 December 2012; accepted 01 March 2014

\begin{abstract}
Considering some complex multi-criteria decision making (MCDM) problems, in which decision environment is dynamic, there are various interdependences among criteria, and plans (systematized alternatives) consisting of multiple time sequential interdependent actions, cannot be well handled by means of the existing MCDM methods, therefore, we develop a systematic decision making (SDM) as an improvement and supplement of the classic MCDM in this paper. The SDM is for prescribing methods of evaluating and selecting the most favourite plan (a system) from a group of feasible ones concerning influences of time-varying criteria system under dynamic external environment. Through detailed analysis, we separate a SDM problem into multi-period MCDM subproblems, and then a plan can be a combination of time sequential strategies in which each strategy (a subset of actions) is a feasible decision choice of corresponding MCDM sub-problem. After clarifying variety of interdependences, interactions and interrelationships in the SDM problems, such as criteria-interdependences, action-interdependences, interactions between criteria and criteria system, interactions between actions and strategies, interactions between strategies and plans, interactions between internal environment (criteria system) and external environment, feedbacks from external environment to the corresponding MCDM sub-problems, and interrelationships among MCDM sub-problems and so on, we transform the SDM into multi-period interrelated MCDM model which can be dealt with more easily by using multiple optimization models. At the end of the paper, three typical properties of the SDM are proposed and most of the existing MCDM models are pointed out as special cases of the SDM.
\end{abstract}

Keywords: multi-criteria, decision making, systematic decision making, criteria system, dynamic, interdependence.

JEL Classification: C52, D81.

Corresponding author Xiaohan Yu

E-mail: yua2006@126.com 


\section{Introduction}

Multi-criteria decision making (MCDM), developed in mid-1960's, prescribes ways of evaluating, ranking and selecting the most favourite alternative from a set of available ones which are characterized by multiple and usually conflicting criteria (Koele 1995; Yager, Rybalov 1998; Campanella, Ribeiro 2011). As usual, the MCDM has been a hot topic in decision sciences and systems engineering, and can be applied into a number of practical problems, such as energy planning (Wang et al. 2009, 2016; Ghosh et al. 2016), supply chain selection (Dey et al. 2016; Dweiri et al. 2016), risk management (Malekmohammadi, Rahimi Blouchi 2014; Vahdat et al. 2014), water resources management (Kumar, Pandey 2016; Weng et al. 2010; Calizaya et al. 2010), and so on. Up to now, lots of pioneers have contributed to developments of the MCDM in order to make it more common and suitable to handle practical and complex decision making problems.

The first kind of complex MCDM problems are MCDM ones with interdependent alternatives. As introduced by Rajabi et al. (1998), multi-criteria subset selection problems are a kind of special MCDM ones, in which each alternative is a subset of actions, i.e., each alternative consists of several actions and there may be one or more mutual actions in different alternatives. In addition, according to Rajabi et al. (1998), actions can be interdependent in many actual multi-criteria subset selection problems, which shed new light on alternativeinterdependences in these problems. A typical example can be deciding how to dispose of solid wastes from a metropolitan area, where we consider possible actions including using one or more of a number of potential dumping sites, incineration at one or more locations, introducing by-laws to reduce the amount of waste generated in the first place, plus a range of recycling measures. Criteria for evaluating each action may include cost, infrastructure requirements, environmental risk, political acceptability, and aesthetics. A feasible alternative may consist of a set of actions that are interdependent for one or more criteria. Furthermore, Fan and Feng (2009) have introduced concepts of individual and collaborative criteria, in which individual criteria are the common criteria but collaborative criteria are shared by pairwise alternatives in corresponding MCDM problems. In their contribution, there are interdependences between a pair of alternatives because of collaborative criteria. Their illustrative example is the selection of a team leader, where individual criteria can be leadership, management experience and professional expertise, while collaborative criteria can be communication, knowledge sharing, and temperament compatibility, and so on. The desired team leader should not only be of high individual capability but also good collaborations with other candidates. The latter are measured by evaluating collaborative criteria which are determined by relationships between pairwise candidates.

Moreover in the literature, it is widely recognized that, in many MCDM problems, criteria are interdependent (Sugeno 1974; Murofushi, Sugeno 1989; Angilella et al. 2004; Xu 2010; Carlsson, Fullèr 1995; Antuchevičiene et al. 2010; Yu, Xu 2012, 2013; Yager 2004, 2008; Yan et al. 2011; da Costa Pereira et al. 2011). A common kind of criteria-interdependences is correlations among criteria. A simple case can be that a tall person is constantly heavy. Thus, if we evaluate a person by taking his height and weight into considerations, the correlation between the two criteria should be noticed. In 1974, fuzzy measure, which can be used to quantify correlations among criteria, has been introduced by Sugeno (1974) 
in his PhD thesis, and meanwhile fuzzy integrals, including Sugeno integral (Sugeno 1974) and Choquet integral (Murofushi, Sugeno 1989), has been verified as a good tool to handle the MCDM problems with correlated criteria together with the fuzzy measure (Angilella et al. 2004; Xu 2010). Some other methods are also used to cope with correlated criteria. Carlsson and Fullèr (1995) has defined a constant to express the degree of correlations among criteria, representing their potential conflict or support. The Manalanobis distance, which takes into account the correlations among criteria, has been integrated by Antuchevičiene et al. (2010) into the model of Technique for the Order Preference by Similarity to Ideal Solution (TOPSIS) instead of the Euclidean distance as an improved method to deal with the MCDM problems with correlated criteria. In addition, a graph has been introduced by $\mathrm{Yu}$ and $\mathrm{Xu}$ (2012) to intuitively represent the correlations among criteria, in which the weight of an arc in the graph can be a quantification of corresponding correlation, and then the MCDM problems with the correlated criteria can be handled. Another kind of criteria-interdependences is prioritizations among criteria (Yager 2004, 2008; Yu and Xu 2013; Yan et al. 2011; Chen, Xu 2015). Yager (2008) has considered a situation in which we are selecting a bicycle for our child based upon the criteria of safety and cost. In this situation we may not allow a benefit with respect to cost to compensate for a loss in safety. Here prioritization is existent between the criteria "safety" and "cost", and safety has a higher priority. Although the kind of criteria-interdependences has just been proposed in recent years, the corresponding problems have been validated to be existent and corresponding methods have been perfectly applied into the problems of information retrieval (da Costa Pereira et al. 2011; Verma, Sharma 2016), preference voting (Amin, Sadeghi 2010), threat evaluation of aerial targets (Huang et al. 2010) and target type recognition (Xu et al. 2010). At present, almost all relevant researchers contribute themselves to how to aggregate evaluating information of multiple criteria with prioritizations among them. We shall pay our attention to the development of methods for handling MCDM problems with prioritized criteria.

Dynamic MCDM problems are complex. First, the explanations of dynamics are in various ways. From the viewpoint of Kornbluth (1992), the changes in a so-called decision making domain, induced by the external conditions/environment, the outcomes of previous decisions, and the decision maker's competitive position, etc., can affect the decision maker's preferences over criteria, and then the importance weights of criteria, thus the dynamics is essentially due to the time-varying weights of criteria which is similar to the opinion of Townsend and Busemeyer (1995). However Townsend and Busemeyer (1995) have focused on a (perhaps long-term) deliberation process of the decision maker, during which the attitudes of the decision maker's preferences over criteria are susceptible by changes of some external conditions and the decision maker's emotion. Liao (1998) have introduced a systematic model to support a kind of complex decision making problems, in which the decision maker has to tackle a serial of decision making tasks, and there is a special relationship, called chain effect, between two sequential decision making tasks (in other words, the decision choice resulting from a previous decision making task affects its subsequent one). The dynamics, in Liao's opinion, originates from time sequential decision making tasks, because of which we must take into account the influences of environments at different moments. Considering a kind of dynamic MCDM problems that the final deci- 
sion is taken only at the end of some exploratory process, Campanella and Ribeiro (2011) have explained the dynamics as that the choices of the decision maker always sway owing to the changes of external conditions and environment, but the final decision always can be made, which resembles an oscillating mechanical spring. In the dynamic model (Campanella, Ribeiro 2011), both alternatives and criteria may vary during dynamic MCDM, and earlier decisions affect later ones differing from the chain effects in the paper of Liao (1998) that a decision only affects the subsequent one.

However, there exist more complex MCDM problems beyond the ability of existing methods. For example, the problems of fire control and assignment. Suppose that we make an operational plan to assign our weapons to some targets in order to reduce the rival's operational effectiveness, we always want to select the best plan from all feasible plans by evaluating the damages of all targets. In this case, the problems of fire control and assignment are MCDM ones, where the plans and targets are alternatives and criteria in the MCDM respectively. Firstly, the plan, which consists of multiple time sequential actions such as the $i^{\text {th }}$ weapon is assigned to the $k^{\text {th }}$ target at moment $t$, is temporal. There may be relationship between a pair of actions. A common relationship is that when we assign the $i^{\text {th }}$ and $j^{\text {th }}$ weapons to an identical target, the situation of first the $i^{\text {th }}$ weapon and then the $j^{\text {th }}$ one is generally different from that of first the $j^{\text {th }}$ weapon and then the $i^{\text {th }}$ one. Thus alternatives in this kind of MCDM are more complicated than those in any one MCDM mentioned above. Secondly, there are varieties of interdependences among targets. A possible interdependence is the cooperation in this kind of problems. We assume that the targets are communication centres in the battlefield, and we aim at paralyzing the rival's communication network. It is needless to damage all communication centres but partial main ones because the communication centres are cooperated. Assistance or support is another kind of interdependence, just as vulnerability of one target will be reduced because of damage of another target. In a concrete fire control and assignment problem, the interdependences among targets (criteria) can be various, which is distinguished from just one kind of interdependences (correlations or prioritizations) in existing models aforementioned. In this sense, fire control and assignment problems are complex enough to existing MCDM models. Besides, similar complex MCDM problems are widely existent in our real-lives: development planning of a corporation (an enterprise, a college or a government), research and development decision making in a technology-based firm, portfolio analysis, and effectiveness evaluation of dynamic tactical communication network in a complex battlefield, etc. These complex MCDM problems cannot be dealt with by existing MCDM methods, thus a systematic decision making model, aiming at the above complex MCDM problems, is developed in this paper as an improvement and supplement of existing MCDM models.

The rest of the paper is organized as follows. In Section 1, we briefly review the classic MCDM and a kind of its general proscess associated with aggregation functions. The scenario of the SDM is stated in Section 2, in which three characteristic terminologies are presented in detail. Then, we propose mathematical representations of the SDM based on optimization models and describe a general framework for dealing with SDM problems in Section 3. Furthermore, some properties are elaborated in Section 4 for the sake of deeper understanding. At length, conclusions are drawn in last Section. 


\section{Multi-criteria decision making (MCDM)}

The classic MCDM prescribes how to select the most favorable alternative from a set of feasible ones which are characterized by multiple, usually conflicting, criteria (Koele 1995; Yager, Rybalov 1998). The fundamental components of a MCDM is a set of criteria, $C=$ $\left\{c_{1}, c_{2}, \ldots, c_{n}\right\}$, of interest to the decision maker and a set of feasible alternatives (decision variables), $X$, which is a subset of a space of decision variables $\mathbf{X}$ (i.e., $X \subseteq \mathbf{X}$ ). In their pioneering work on MCDM, Bellman and Zadeh (1970) have suggested that each criterion $c_{i}$ $\in C$ can be represented as a fuzzy subset over the decision variables in $\mathbf{X}$. In particular, for a criterion $c_{i} \in C$ we can represent it as a fuzzy subset $c_{i}$ over $\mathbf{X}$ such that $c_{i}(x) \in[0,1]$ is the degree to which this criterion is satisfied by a decision variable $x \in \mathbf{X}$. Then essentially, the underlying MCDM model can be formulated as a multi-objective or vector optimization problem (Kaliszewski et al. 2012):

$$
\begin{aligned}
& \max _{i \in\{1,2, \ldots, n\}} c_{i}(x) \\
& \text { s.t. } \quad x \in X,
\end{aligned}
$$

which means a feasible decision variable is the most favourite if it can satisfy the decision maker to an extreme with respect to all criteria. However, due to conflicts among criteria in most of MCDM problems, it is usually impossible to pick out a decision variable $x$ from $X$ such that it has the highest satisfaction degree with respect to every criterion. Thus some rules over criteria, such as distinguishing importance weights of criteria, are introduced so as to transform the above multi-objective optimization problem to single-objective one. A common transformation is based on aggregation function defined as:

Definition 1 (Grabisch et al. 2009). An aggregation function is a mapping $A F:[0,1]^{n} \rightarrow[0,1]$, whenever it is non-decreasing, $A F(0,0, \ldots, 0)=0$ and $A F(1,1, \ldots, 1)=1$.

In accordance with potential rules in a MCDM problem, an aggregation function can be designed to integrate satisfaction degrees of a decision variable $x$ with respect to all criteria into an overall one, i.e.,

$$
c(x) \quad A F\left(c_{1}(x), c_{2}(x), \ldots, c_{n}(x) .\right.
$$

In this case, the multi-objective optimization model in can be simplified as a singleobjective one:

$$
\begin{aligned}
& \max \quad c(x) \quad A F\left(c_{1}(x), c_{2}(x), \ldots, c_{n}(x)\right. \\
& \text { s.t. } x \in X .
\end{aligned}
$$

Here by, we attempt to derive the most favourite $x$ with the maximal overall satisfaction degree from $X$.

From the above analysis, the generalized process of the MCDM based upon the aggregation function is as follows (see Fig. 1):

1) Anatomize a decision making problem, and extract its decision objects (i.e., feasible decision variables/alternatives mentioned above). Determine the set of feasible alternatives and the set of criteria. 


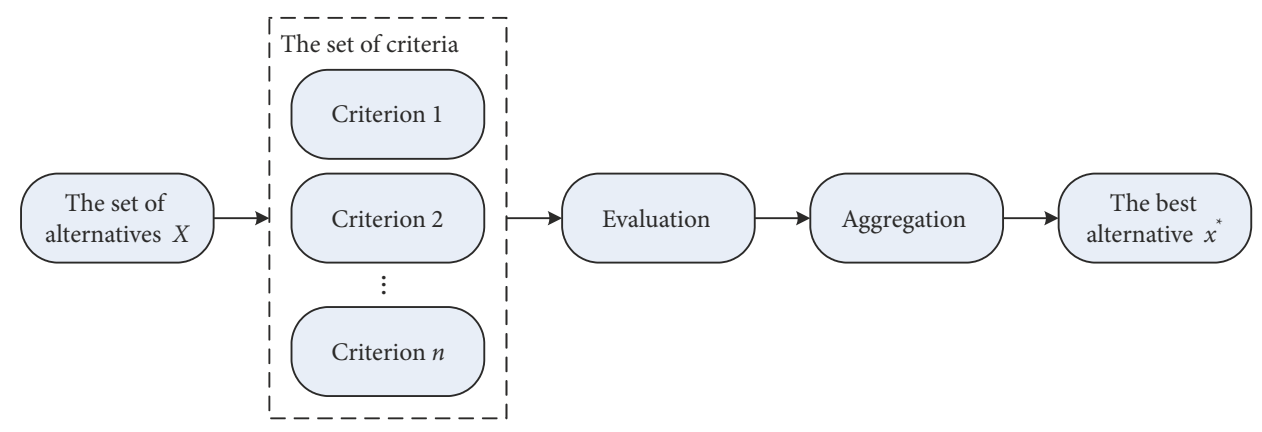

Fig. 1. The process of MCDM

2) Evaluate each alternative with respect to each criterion. Original evaluating information may be in various forms, such as real numbers, fuzzy numbers, interval values, fuzzy subsets and linguistic variables, etc. Thus in this step, we shall normalize all evaluating information into satisfaction degrees, i.e., membership degrees of fuzzy subsets just like $c_{i}(x)$ in (1).

3) In accordance with the characteristic of the MCDM problem, choose a suitable aggregation function, by means of which all satisfaction degrees of each alternative are integrated into an overall one.

4) At length, we select the best alternative(s) on the basis of their overall satisfaction degrees. If it is necessary, we rank all alternatives.

\section{Scenario of systematic decision making (SDM)}

In the classic multi-criteria decision making (MCDM) model, we usually evaluate, rank and select the most favourite one from a group of isolated and static alternatives based on multiple independent and conflicting criteria. However many complex MCDM problems, such as fire control and assignment problems, cannot be modeled by the classic MCDM, because of their various criteria-interdependences and complicated forms of alternatives. In the sense, the complex MCDM can be constructed as a systematic decision making (SDM) which involves at least: a) a criteria system, b) plans (systematized alternatives), and c) an external environment. Essentially, the SDM is used to prescribe a kind of processes that evaluate and select the optimal plan from all feasible plans on the basis of a criteria system.

\subsection{Criteria system}

In the SDM problems, there are usually various interdependences among criteria, which greatly affect decision choices, so we must take into account both criteria and interdependences among them. In the situation that we want to paralyze the rival's communication network, the best strategy must be to damage all communication centres if interdependences among these communication centres are ignored, whereas in fact, perhaps just half connected communication centres need to be damaged. There is diversity of criteria-inter- 
dependences. The first one is influence, which means one criterion can influence the other's evaluation, if there is influence between the pair of criteria, but the latter cannot influence the former. For example, early warning aircrafts can strengthen threats of relevant fighter aircrafts in a fire control and assignment problem. If two or more criteria can influence each other, correlations will be existent among them. Fighter aircrafts are correlated in an effectual formation, because the threats of aircrafts are strengthened with the help of others. Besides, prioritizations may exist in some SDM problems. We usually partition targets into several levels in a combat concerning the targets' affections to entire battle. In the case, the targets in a higher level are of higher prioritizations than those in a lower level. According to Yager $(2004,2008)$, the loss of a criterion with higher prioritization cannot be compensated by benefits of criteria with lower prioritizations. Criteria-interdependences are usually specific in the concrete SDM problems, thus we shall describe them properly in accordance with the distinctiveness of the problems.

In the SDM, multiple criteria and all interdependences among them constitute a system, called a criteria system in this paper, in which each criterion is an element of the criteria system and the structure of the criteria system consists of all criteria-interdependences. When evaluating a plan in a SDM problem, we must be based on the holistic criteria system but not respective criteria. The criteria system is usually regarded as the internal environment of a SDM problem. The internal environment is usually time-varying and dynamic, in other words, during the process of the SDM, criteria and criteria-interdependences may vary. As mentioned in the section of Introduction, targets are criteria of the corresponding SDM in a fire control and assignment problem. At all times, some targets may be damaged, or some new targets may arrive, which means we must pay attention to the changes of the criteria systems when handling a SDM problem just like the fire control and assignment problem.

\subsection{Plans: systematized alternatives}

Similar to alternatives in the MCDM, we need to evaluate some plans so as to select the best one(s) in the SDM. A plan consists of multiple time sequential strategies differing from an alternative. A strategy is usually regarded as a decision choice of a static MCDM problem (a sub-problem of the SDM problem). In this case, a SDM problem can be described as a combination of a serial of static MCDM sub-problems. We can derive strategies by dealing with all MCDM sub-problems and synthesis them into a plan of the SDM problem. For example, in such a SDM problem that we assign $m$ weapons to $n$ targets in order to reduce the rival's operational effectiveness as much as possible, the entire combat usually has multiple periods and during each period each weapon is assigned into one target according to launch traits of weapons. Such a SDM problem can be divided into multi-period MCDM sub-problems. During a period, the corresponding MCDM sub-problem can be considered to be static, in which we can devise feasible strategies like a strategy is to assign the $i^{\text {th }}$ weapon to the $k^{\text {th }}$ target, the $j^{\text {th }}$ weapon to the $l^{\text {th }}$ target, etc. We finally assemble strategies of sub-problems during different periods into a feasible plan.

A point we must notice is that there may be interrelationships among these MCDM sub-problems, in which two kinds of typical interrelationships are relations of cause and 
effect and anti-causalities. The former is described as outcomes of the earlier MCDM subproblems affect later ones. If a target is damaged during a period, we will exclude it from the criteria system in subsequent sub-problems, which means outcomes of earlier subproblems can affect the internal environment of later ones. Absolutely, outcomes of earlier sub-problems also can affect the external environment of later ones, as terrains, roads and bridges etc., are easily changed by artillery fire. Finally, decision choices of later sub-problems will be affected. Anti-causality means the possible condition of the criteria system in a future sub-problem affects decision choice at present. A typical example is the use of missiles in the battlefield. As we know, missiles are ammunitions with high effectiveness, and usually can fulfill combat missions within a short time. However, we must use missiles with restraint, because we need to prevent a situation that missiles are used up when we are in urgent need of them. Because of the existence of interrelationships among MCDM sub-problems, time sequential strategies are not independent any more, which is a complex case of the SDM problems.

A strategy can be a subset of actions, which is contained in the set of all possible actions. An action in the fire control and assignment problem can be the $i^{\text {th }}$ weapon is assigned to the $k^{\text {th }}$ target. As soon as a target is in the range of a weapon, assign the weapon to the target will be a possible action. There also may be interdependences among actions. A common kind of action- interdependences is that when we assign the $i^{\text {th }}$ and $j^{\text {th }}$ weapons to an identical target, the situation of first the $i^{\text {th }}$ weapon and then the $j^{\text {th }}$ one is generally different from that of first the $j^{\text {th }}$ weapon and then the $i^{\text {th }}$ one, which is temporal actioninterdependences. Another kind of action-interdependences is non-temporal, just like the efficiency of compound attacks of two or more weapons is usually more than the summation of respective attack efficiencies of these weapons.

A plan can be regarded as a system in the SDM, in which an action is an element of the system and a strategy is a sub-system. Essentially, a plan is an assemblage of actions involved and corresponding action-interdependences. Strategies and corresponding MCDM sub-problems of a SDM problem are just intermediates during the SDM. Because of the complexity of some SDM problems, we generally take some simplifications by dividing them into the multiple MCDM sub-problems. There may be constantly various partition ways, thus the serials of sub-problems are flexible. Besides, forms of strategies are accordance with the corresponding sub-problems. From the above analysis, we conclude that the SDM is actually a model that evaluate and select the most favourite system (plan) from a group of plans which is characterized by criteria system, which is why we call such a decision making model the systematic decision making.

\subsection{External environment}

There seldom is description about external environment in the MCDM, because the static and invariable external environment of the MCDM is constantly neglected during the period of decision making. However, the interaction between the criteria system and its external environment constantly occurs from the viewpoint of system theory. Inconstant 
external environment always affects some decision factors of the SDM tremendously including the structure of the criteria system and evaluating information etc., and finally the decision choices. Generally speaking, there are two kinds of external environments: a) Inherent environment, whose dynamics is inherent but generally uncertain and hard to be affected by the decision choice of earlier MCDM sub-problems, is the first kind of external environment, such as the wind, the brightness and the air temperature, etc. of the battlefield in a fire control and assignment problem; b) The other kind is called coherent environment, which means the coherent environment's changes are related to the decision choices of the previous MCDM sub-problems. For instance, the environment of communication channels is easily interfered, and even some new type meteorological weapons can change the weather. We must make it clear that the gap between the inherent and coherent environment is vague, because an inherent environment in a SDM problem may be a coherent one in another problem, and vice versa.

The influences from the coherent environment to the structure of the criteria system or the evaluating information is called feedbacks in this paper, which means earlier influences from decision choices to the coherent environment are fed back to later MCDM subproblems. Feedbacks are essentially a special kind of relations of cause and effect, which will greatly affect the selection of the optimal plan.

\section{Model architecture of systematic decision making (SDM)}

A generalized mathematic representation of the SDM can be an optimization model:

$$
\begin{aligned}
& \max F_{\mathbf{C}}(p) \\
& \text { s.t. } p \in P,
\end{aligned}
$$

where $P$ denotes the set of all feasible plans, and $F_{\mathbf{C}}(p)$ means the satisfaction degree of a feasible plan $p$ with respect to the criteria system $\mathbf{C}$. The larger $F_{\mathbf{C}}(p)$, the better the plan $p$ with respect to $\mathbf{C}$, which means we find out the optimal plan $p^{*}$ from the set of feasible plans $P$ by taking influences of the criteria system $\mathbf{C}$ into consideration (see Fig. 2).

Contrast to the multi-criteria decision making (MCDM) in Section 1, the model in is similar to except that the objective function in is a function concerning the entire criteria system, while that in is an aggregation function with respect to the set of criteria in the

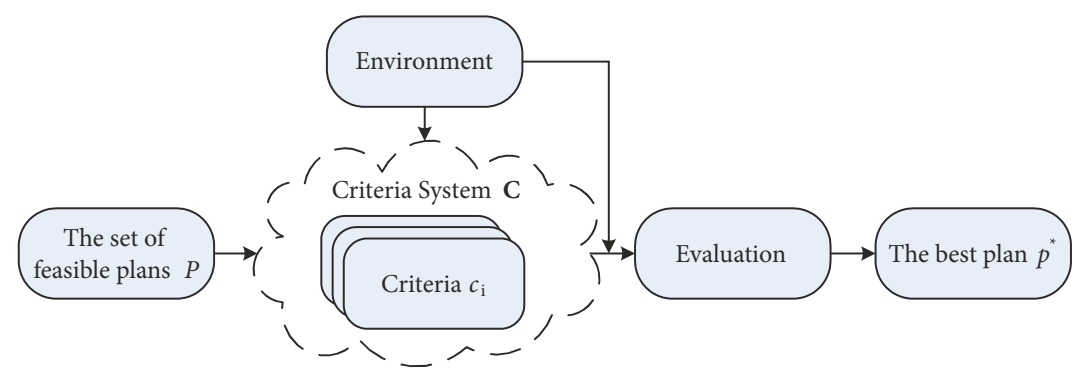

Fig. 2. The framework of the SDM 
MCDM. We cannot simply regard the objective function in as a function just associated with criteria in the criteria system, because the criteria system is not just an assemblage of criteria but also including corresponding criteria-interdependences. Besides, differing from the framework of the MCDM in Figure 1, we cannot ignore the influence of time-varying external environment to the structure of the criteria and the evaluations of plans during the process of the SDM. Furthermore, a complex systematized plan is hard to be straightly evaluated based on some criteria let alone the criteria system. Thus we need to simplify the model in.

The first step is to divide the SDM problem involved into a serial of time sequential MCDM sub-problems as mentioned in the preceding. In each sub-problem, the criteria system is static and the external environment can be considered to be invariable, based on which we evaluate some strategies and select the best one. Suppose that a SDM problem is divided into $t$ MCDM sub-problems corresponding to $t$ periods $\left(T_{1}, T_{2}, \ldots, T_{t}\right)$ respectively, and each plan $p$ of the SDM problem is then the combination of $t$ strategies, i.e., $p=\left(s_{1}, s_{2}, \ldots, s_{k}, \ldots, s_{t}\right)$. In this case, if we derive feasible strategies aiming at the $t$ sub-problems, a feasible plan will be obtained. Moreover, if these strategies are optimal in respective sub-problems concerning their interrelationships, the optimal plan can be constructed by these optimal strategies. Therefore, we must firstly deal with $t$ interrelated optimization problems in order to derive the optimal strategies for the respective MCDM sub-problems:

$$
\begin{aligned}
& \max _{k=1, \ldots, t} F_{\mathbf{C}_{k}}\left(s_{k}\right) \\
& \text { s.t. } \quad s_{k} \in S_{k},
\end{aligned}
$$

where $S_{k}$ denotes the set of feasible strategies during the period $T_{k}, \mathbf{C}_{k}$ denotes the criteria system during the period $T_{k}$, and $F_{\mathbf{C}_{k}}\left(s_{k}\right)$ means the satisfaction degree of a feasible strategy $s_{k}$ with respect to $\mathrm{C}_{k}$. Corresponding processes of the model can refer to Figure 3 .

In Figure 3, we need to deal with a MCDM sub-problem during each period of $T_{k}$ $(k=1,2, \ldots, t)$ so as to select the best strategy $s_{k}^{*}$ from $S_{k}$ based on a criteria system $\mathbf{C}_{k}$ with determined criteria and criteria-interdependences under the influence (feedback) of current external environment. Before that, we have to initial the set of feasible strategies $S_{k}$ $(k=1,2, \ldots, t)$, the external and internal environment during this period $T_{k}$ by considering interrelationships (relations of cause and effect, and anti-causalities) among MCDM subproblems during different periods. Decision choices of earlier MCDM sub-problems affect the set of feasible strategies, the external and internal (criteria system) environment of later ones, which is described as relations of cause and effect (directed dotted lines in Fig. 3). Whereas the anti-causalities (directed thick lines in Fig. 3) are mainly that the internal environment (criteria system) during subsequent periods may change optional strategies of current sub-problem.

However, the overall satisfaction degree $F_{\mathbf{C}_{k}}\left(s_{k}\right)$ of a strategy $s_{k}$ with respect to $\mathbf{C}_{k}$ in is usually far harder to be directly obtained than the satisfaction degree $c_{i}\left(s_{k}\right)$ of $s_{k}$ with respect to a criterion $c_{i}$ in the system $\mathbf{C}_{k}$, thus we usually aggregate all satisfaction degrees with respect to criteria into the overall one $F_{\mathbf{C}_{k}}\left(s_{k}\right)$ concerning criteria-interdependences synchronously by using some function (similar to the aggregation functions in the MCDM). 


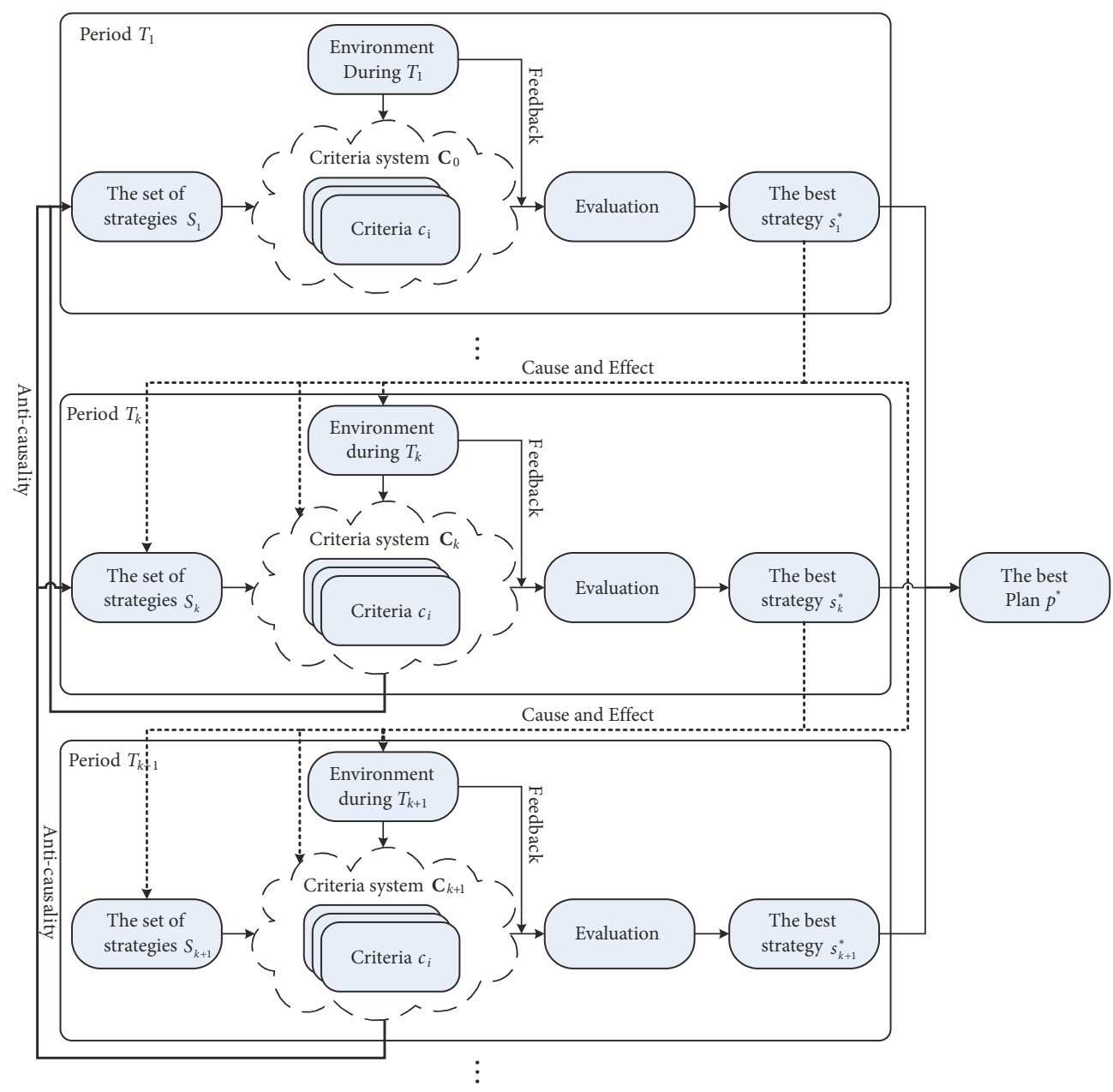

Fig. 3. The processes of the SDM

Good precedents can be Choquet integral associated with fuzzy measures (Sugeno 1974; Murofushi, Sugeno 1989; Angilella et al. 2004; Xu 2010) and prioritized aggregation operators (Yager 2004, 2008; Yu, Xu 2013; Yan et al. 2011), which successfully aggregate satisfaction degrees with respect to criteria into the overall one synchronously concerning correlations and prioritizations among criteria respectively. Accordingly in what follows, we devise a new kind of aggregation functions for the criteria system.

In accordance with the idea of the graph theory, we partition a criteria system $\mathbf{C}$ into two sets: the set of criteria $C$ and the set of criteria-interdependences $R$ in the system, denoted as $\mathbf{C}=(C, R)$. Especially during the period $T_{k}$, we have $\mathbf{C}_{k}=\left(C_{k}, R_{k}\right)$, and the overall satisfaction degree of $s_{k}$ can be rewritten as $F_{\mathbf{C}_{k}}\left(s_{k}\right)=F_{C_{k}, R_{k}}\left(s_{k}\right)$. If a function $A \tilde{F}$ is a mapping from $[0,1]^{n}$ to $[0,1]$ satisfying $A \tilde{F}(0,0, \ldots, 0)=0$, we call $A \tilde{F}$ a systematic aggregation function, where $A \tilde{F}(0,0, \ldots, 0)=0$ means if all elements do nothing in a system, 
their entirety will also do nothing. We must notice that the systematic aggregation functions do not satisfy non-decreasing monotonicity differing from the aggregation function in Definition 1, which is because the holistic emergence of a system affects its holistic effectiveness. For example, a criterion negatively affects benefits of other criteria in a criteria system. Increasing the satisfaction degree of the criterion will reduce the overall satisfaction degree of the criteria system, if satisfaction degrees of other criteria keep unchanged.

Suppose there is a criteria system $\mathbf{C}_{k}=\left(C_{k}, R_{k}\right)$ with $n$ criteria, based on a systematic aggregation function $A \tilde{F}$, we have $F_{\mathrm{C}_{k}}\left(s_{k}\right)=A \tilde{F}_{R_{k}}\left(c_{1}\left(s_{k}\right), c_{2}\left(s_{k}\right), \ldots, c_{n}\left(s_{k}\right)\right)$, where $A \tilde{F}_{R_{k}}$ denotes a systematic aggregation function associated with the set of criteria-interdependences $R_{k}$. In this case, the model in can be rewritten as:

$$
\begin{aligned}
& \max _{k=1, \ldots, t} F_{\mathbf{C}_{k}}\left(s_{k}\right)=A \tilde{F}_{R_{k}}\left(c_{1}\left(s_{k}\right), c_{2}\left(s_{k}\right), \ldots, c_{n}\left(s_{k}\right)\right) \\
& \text { s.t. } \quad s_{k} \in S_{k} .
\end{aligned}
$$

\section{Properties of systematic decision making (SDM)}

The SDM in this paper is a generalized multi-criteria decision making (MCDM) model, and most existing MCDM models are its special cases:

a) If there is only a MCDM sub-problem in which strategies are the subsets of one action and there is no any criteria-interdependence, the corresponding SDM will reduce to a classic MCDM model described in Section 1.

b) If there is only a MCDM sub-problem in which there is no any criteria-interdependence, the corresponding SDM will reduce to a multi-criteria subsets decision making model (Rajabi et al. 1998).

c) If there is only a MCDM sub-problem in which strategies are the subsets of one action and there are just correlations among criteria, the corresponding SDM will reduce to a MCDM model with the correlated criteria (Sugeno 1974; Murofushi, Sugeno 1989; Angilella et al. 2004; Xu 2010; Carlsson, Fullèr 1995; Antuchevičiene et al. 2010; Yu, Xu 2012).

d) If there is only a MCDM sub-problem in which strategies are the subsets of one action and there are just prioritizations among criteria, the corresponding SDM will reduce to a prioritized MCDM model (Yager 2004, 2008; Yu, Xu 2013; Yan et al. 2011).

e) If there are a serial of MCDM sub-problems in which strategies are the subsets of one action, there is no any criteria-interdependence, and the anti-causalities are not existent among the sub-problems, the corresponding SDM will reduce to the dynamic MCDM model introduced by Campanella, Ribeiro (2011).

In addition, we discuss three properties of the SDM: wholeness, character of hierarchy, and dynamic nature, so as to further elaborate the SDM in this section. 


\subsection{Wholeness}

There are two explanations about the wholeness of the SDM:

a) The criteria system is an entirety, but not just an assemblage of several independent criteria. Thus when evaluating a plan, we had better to be based on the entire criteria system rather than one or several criteria concerned.

b) Any plan is holistic. In the last section, we divide a SDM problem into a serial of MCDM sub-problems, and because of this we can evaluate and select the best strategy for each sub-problem as the intermediate of the SDM problem. We must notice that a plan, consisting of all optimal strategies, commonly is not the optimal plan of the SDM problem, if interrelationships among the sub-problems are neglected.

Holistic emergence is the manifestation of the SDM's wholeness. Two groups of targets, with identical objects but different interdependences among them, are attacked by one operational plan. The results may be dramatically different, because of the distinguished holistic emergences of these two criteria systems. The other kind of holistic emergences are about plans. Relevant example is the optimal operational plan which is usually not the one with the maximal consumptions of weapons but that utilizes the interactions among weapons most properly.

Due to the wholeness of the SDM, it concludes that any practical method of the SDM is a combined method of holism and reductionism. As soon as analyzing actions based on criteria, we must holistically grasp relevant plans concerning the criteria system. Only in this way can we make sure outcomes of the model in are consistent with the model in.

\subsection{Character of hierarchy}

A criteria system is hierarchical, which means a criterion may be also a system and a criteria system may be an element of a broader criteria system. Just like in a digitized force, each combat unit can be a system: tanks, artilleries and helicopters consist of mechanical parts, and soldiers consist of organs, but during a joint operation, the digitized force is just a criterion of joint forces. In addition, we must make it clear which hierarchy is focused on in a SDM problem concerned. For example, if we make an operational plan against the rival's tanks, threats of these tanks are desired. In this case, it is not necessary to analyze the emergence of each tank's parts in detail, but take some holistic analysis, such as statistical analysis, so as to obtain an approximate threat. However, if we just want to evaluate the threat of a single tank, it is necessary to analyze all relevant parts and their emergence, because the threat of the tank is essentially an emergence of its parts.

Plans are hierarchical. As mentioned in Subsection 2.2, a plan is a system which consists of multiple interdependent actions. Meanwhile, between hierarchies of plan and action, there is a hierarchy of strategy. A strategy, including some actions, is a subsystem of the corresponding plan. Introduction of strategies results from partitioning the corresponding SDM problem into a serial of time sequential MCDM sub-problems. Therefore, the partition of a plan is unfixed. 


\subsection{Dynamic nature}

Generally speaking, most SDM problems are long-term or time-dependent, in which we evaluate and select the most favourite plan from a group of time sequential plans. That is the primary reason that the SDM is dynamic.

Firstly, the external environment is time-varying. A SDM problem is usually divided into multi-period MCDM sub-problems. Although the external environment is considered to be stable and unchanged during a period (so is the internal environment), we must notice differences of the external environment among different sub-problems, and initial the external environment of each sub-problem as precondition before handling the subproblem. In addition, except for the inherent and coherent environment mentioned in Subsection 2.3, we had better pay attention to effects of the rival's or competitor's decision choices to the external environment if it is necessary.

Secondly, susceptible decision maker's preferences are noteworthy. In the SDM, decision maker's preferences can affect the importance weights of criteria and even some criteria-interdependences. For example, the commander may change the level of targets during a fire control and assignment problem, which will affect prioritizations among targets (criteria).

The third is the adjustment of criteria system (internal environment). We must become aware of the change of criteria system especially the change of its structure even though delicate change, because the delicate change may result in great variance of decision choices due to the emergence of the criteria system.

\section{Illustrative example}

In this section, we will take a simple fire control example to elaborate the process of the systematic decision making (SDM).

Suppose that we have three kinds of weapons, $a_{1}, a_{2}$ and $a_{3}$, aiming at damage several targets which arrive in batches, where there are $n_{1}=5, n_{2}=4$ and $n_{3}=6$ ammunitions for $a_{1}, a_{2}$ and $a_{3}$ respectively. In this fire control problem, we must assign the ammunitions of weapons to respective targets according to the devised optimal fire assignment strategy when a batch of targets arrive, but we have no idea the number and type of the next batch at this moment. In this problem, we assume there are two batches of targets, $\left(b_{1}, b_{2}\right)$ and $\left(b_{3}\right)$, where $b_{j}(j=1,2,3)$ denote targets. Besides, some information has already been given as follows:

1) The surviving probabilities $p_{i j}(i=1,2,3 ; j=1,2,3)$ that one ammunition of $a_{i}$ damages $b_{j}$ have been given, and all probabilities consist of a probability matrix:

$$
P=\left(p_{i j}\right)_{3 \times 4}=\left(\begin{array}{lll}
0.90 & 0.80 & 0.51 \\
0.85 & 0.49 & 0.67 \\
0.95 & 0.62 & 0.68
\end{array}\right)
$$


According to $p_{i j}$, we can calculate the surviving probability of any target under a certain fire assignment strategy. For instance, if two ammunitions of $a_{1}$ and one of $a_{2}$ are assigned to $b_{1}$, the surviving probability of $b_{1}$ is then $p_{11}^{2} \cdot p_{21}^{1}=0.90^{2} \times 0.85^{1}=0.689$.

2) There may exist joint damages when two weapons attack one target:

- If an ammunition of $a_{1}$ and one of $a_{2}$ are jointly assigned to damage $b_{2}$, the surviving probability of $b_{2}$ is set as $\left(p_{12} \cdot p_{22}\right)^{1.5}=0.245$ in this problem.

- If an ammunition of $a_{1}$ and one of $a_{3}$ are jointly assigned to damage $b_{1}$, the surviving probability of $b_{1}$ is set as $\left(p_{11} \cdot p_{31}\right)^{1.5}=0.791$.

- If an ammunition of $a_{2}$ and one of $a_{3}$ are jointly assigned to damage $b_{3}$, the surviving probability of $b_{3}$ is set as $\left(p_{23} \cdot p_{33}\right)^{1.5}=0.308$.

3) There are connections among targets:

- The targets can be partitioned into two prioritized levels, $\left\{b_{1}\right\} \succ\left\{b_{2}, b_{3}\right\}$, according to combat mission, and if $b_{1}$ were damaged, it would be more likely for us to accomplish the mission and win the combat.

- There exists cooperation relationship between $b_{2}$ and $b_{3}$, i.e., if both $b_{2}$ and $b_{3}$ are survival, their threats will be double.

A fire assignment strategy can be a matrix $S=\left(s_{i j}\right)$, where $s_{i j}$ means the ammunition number of $a_{i}$ being assigned to $b_{j}$. In the situation that the $1^{\text {st }}$ batch of targets $\left(b_{1}\right.$ and $\left.b_{2}\right)$ arrive, a fire assignment strategy can be $S^{(1)}=\left(s_{i j}^{(1)}\right)_{3 \times 2}$. After taking into account the above joint damages, surviving probabilities of $b_{1}$ and $b_{2}$ can then be derived by:

$$
\begin{aligned}
& p\left(b_{1}\right)=\left(\left(p_{11} \cdot p_{31}\right)^{1.5}\right)^{\min \left(s_{11}^{(1)}, s_{31}^{(1)}\right)} \cdot p_{11}^{s_{11}^{(1)}-\min \left(s_{11}^{(1)}, s_{31}^{(1)}\right)} \cdot p_{21}^{s_{21}^{(1)}} \cdot p_{31}^{s_{31}^{(1)}-\min \left(s_{11}^{(1)}, s_{31}^{(1)}\right)}= \\
& p_{11}^{s_{11}^{(1)}+0.5 \cdot \min \left(s_{11}^{(1)} s_{31}^{(1)}\right)} \cdot p_{21}^{s_{21}^{(1)}} \cdot p_{31}^{s_{31}^{(1)}+0.5 \cdot \min \left(s_{11}^{(1)}, s_{31}^{(1)}\right)}
\end{aligned}
$$

and

$$
p\left(b_{2}\right)=p_{12}^{s_{12}^{(1)}+0.5 \cdot \min \left(s_{12}^{(1)}, s_{22}^{(1)}\right)} \cdot p_{22}^{s_{22}^{(1)}+0.5 \cdot \min \left(s_{12}^{(1)}, s_{22}^{(1)}\right)} \cdot p_{32}^{s_{32}^{(1)}}
$$

In this case, our purpose is to minimize surviving probabilities of all targets by selecting the optimal fire assignment strategy in the fire control problem, which actually is an SDM problem where each target is a criteria (evaluated by the surviving probability), and a fire assignment strategy is a strategy consisting of several actions (an action is some ammunitions of a weapon are assigned to a target).

Sequentially, we model and handle the fire control problem based on the SDM.

When the $1^{\text {st }}$ batch of targets $\left(b_{1}\right.$ and $\left.b_{2}\right)$ arrives, we need to assign some ammunitions to attach them, and we obtained intelligence that some other targets will arrive sooner or later but their types and numbers are unknown. In such a case, some ammunitions shall be saved for sequential targets, which is just the anti-causality between current decision making and sequential decision making. We simply assume ammunition numbers of $a_{1}$, $a_{2}$ and $a_{3}$, used in current fire assignment, are 3, 2 and 3 respectively, and the rest of ammunitions will be used in the future. We then construct a multi-criteria decision making (MCDM) model to deal with current problem: 


$$
\begin{array}{ll}
\min & F_{1}\left(p\left(b_{1}\right), p\left(b_{2}\right)\right. \\
\text { s.t. } & s_{11}^{(1)}+s_{12}^{(1)} \leq 3 \\
& s_{21}^{(1)}+s_{22}^{(1)} \leq 2 \\
& s_{31}^{(1)}+s_{32}^{(1)} \leq 3 \\
& s_{i j}^{(1)} \in \mathbb{N}_{0}, i=1,2,3 ; j=1,2,
\end{array}
$$

where $\mathbb{N}_{0}$ denotes the set of all non-negative integers. Because there exists prioritization between $b_{1}$ and $b_{2}$, i.e., $b_{1} \succ b_{2}$, we can aggregate $p\left(b_{1}\right)$ and $p\left(b_{2}\right)$ in accordance with Yager (2008) as:

$$
F_{1} p\left(b_{1}\right), p\left(b_{2}\right)=p\left(b_{1}\right)+p\left(b_{1}\right) \cdot p\left(b_{2}\right) .
$$

Therefore the above model can be solved, and the optimal fire assignment strategy of current period can be:

$$
S^{(1) *}=\left(\begin{array}{ll}
s_{11}^{(1) *} & s_{12}^{(1) *} \\
s_{21}^{(1) *} & s_{22}^{(1) *} \\
s_{31}^{(1) *} & s_{32}^{(1) *}
\end{array}\right)=\left(\begin{array}{ll}
2 & 1 \\
1 & 1 \\
2 & 1
\end{array}\right) .
$$

In this case, the surviving probabilities of $b_{1}$ and $b_{2}$ are $p\left(b_{1}\right)=0.531$ and $p\left(b_{2}\right)=0.152$ respectively. There will be four situations when the $2^{\text {nd }}$ batch of target $\left(b_{3}\right)$ arrive: 1$)$ we need to attack all three targets, i.e., both $b_{1}$ and $b_{2}$ are survival after the first round of attacks under the optimal $1^{\text {st }}$ period fire assignment strategy, whose probability is $p\left(b_{1}\right) \times p\left(b_{2}\right)=0.081$; 2) we need to attack $b_{1}$ and $b_{3}$, whose probability is $\left.p\left(b_{1}\right) \times\left(1-p\left(b_{2}\right)\right)=0.450 ; 3\right)$ we need to attack $b_{1}$ and $b_{3}$, whose probability is $\left(1-p\left(b_{1}\right)\right) \times p\left(b_{2}\right)=0.071$; and 4$)$ only $b_{3}$ need to be attacked, i.e., both $b_{1}$ and $b_{2}$ are damaged in this situation, whose probability is 0.398 .

As an explanation, we just discuss the $3^{\text {rd }}$ situation when $b_{3}$ arrives, i.e., $b_{1}$ is damaged but $b_{2}$ is survival after the optimal $1^{\text {st }}$ period fire assignment strategy is carried out. In this situation, we shall devise another MCDM model to handle the $2^{\text {nd }}$ period fire assignment problem:

Similary,

$$
\begin{array}{ll}
\min & F_{2}\left(p\left(b_{2}\right), p\left(b_{3}\right)\right. \\
\text { s.t. } & s_{12}^{(2)}+s_{13}^{(2)} \leq 2 \\
& s_{22}^{(2)}+s_{23}^{(2)} \leq 2 \\
& s_{32}^{(2)}+s_{33}^{(2)} \leq 3 \\
& s_{i j}^{(2)} \in \mathbb{N}_{0}, i=1,2,3 ; j=2,3 .
\end{array}
$$

$$
p\left(b_{2}\right)=p_{12}^{s_{12}^{(2)}+0.5 \cdot \min \left(s_{12}^{(2)}, s_{22}^{(2)}\right)} \cdot p_{22}^{s_{22}^{(2)}+0.5 \cdot \min \left(s_{12}^{(2)}, s_{22}^{(2)}\right)} \cdot p_{32}^{s_{32}^{(2)}}
$$

and

$$
p\left(b_{3}\right)=p_{13}^{s_{13}^{(2)}} \cdot p_{23}^{s_{23}^{(2)}+0.5 \cdot \min \left(s_{23}^{(2)}, s_{33}^{(2)}\right)} \cdot p_{33}^{s_{33}^{(2)}+0.5 \cdot \min \left(s_{23}^{(2)}, s_{33}^{(2)}\right)}
$$

Besides as mentioned above, if both $b_{2}$ and $b_{3}$ are still survival at the end of the $2^{\text {nd }}$ round of attacks, their threats will be double. Thus the objective function can be 
$F_{2}\left(p\left(b_{2}\right), p\left(b_{3}\right)\right)=p\left(b_{2}\right) \cdot p\left(b_{3}\right) \cdot\left[2 \cdot\left(p\left(b_{2}\right)+p\left(b_{3}\right)\right)\right]+\left(1-p\left(b_{2}\right) \cdot p\left(b_{3}\right)\right) \cdot\left(p\left(b_{2}\right)+p\left(b_{3}\right)\right)$

where the first part means the overall threat must be double if both $b_{2}$ and $b_{3}$ are survival, otherwise the overall threat is the second part. We simplify the above expression as:

$$
F_{2}\left(p\left(b_{2}\right), p\left(b_{3}\right)\right)=\left(1+p\left(b_{2}\right) \cdot p\left(b_{3}\right)\right) \cdot\left(p\left(b_{2}\right)+p\left(b_{3}\right)\right) .
$$

We solve the above MCDM model and derive the optimal $2^{\text {nd }}$ period fire assignment strategy:

$$
S^{(2) *}=\left(\begin{array}{ccc}
s_{11}^{(2) *} & s_{12}^{(2) *} & s_{13}^{(2) *} \\
s_{21}^{(2) *} & s_{22}^{(2) *} & s_{23}^{(2) *} \\
s_{31}^{(2) *} & s_{32}^{(2) *} & s_{33}^{(2) *}
\end{array}\right)=\left(\begin{array}{ccc}
0 & 1 & 1 \\
0 & 1 & 1 \\
0 & 2 & 1
\end{array}\right)
$$

In addition, the surviving probabilities of $b_{2}$ and $b_{3}$ are $p\left(b_{2}\right)=0.094$ and $p\left(b_{3}\right)=0.157$ respectively.

Therefore, in the situation that $b_{1}$ is damaged but $b_{2}$ is survival after the $1^{\text {st }}$ round of attacks, the best plan is then the combination of $S^{(1) *}$ and $S^{(2) *}$, i.e., $S^{*}=\left(S^{(1) *}, S^{(2) *}\right)$.

From the above process, we clear that the fire control problem is actually an SDM problem with two MCDM sub-problems. We must first consider the anti-causality of the $2^{\text {nd }}$ MCDM sub-problem (i.e., the fire control problem after $b_{3}$ arrives) in order to get a proper fire assignment strategy. If the anti-causality is ignored, we will assign all ammunitions to $b_{1}$ and $b_{2}$, and there will be no ammunitions to damage $b_{3}$, which is obviously not the best plan. Besides, the strategy of the $1^{\text {st }} \mathrm{MCDM}$ sub-problem will affect the environment of the $2^{\text {nd }}$ MCDM sub-problem (i.e., the cause and effect), including the number of usable ammunitions and surviving probabilities of $b_{1}$ and $b_{2}$. As mentioned above, there are four situations with respect to the $2^{\text {nd }} \mathrm{MCDM}$ sub-problem (the $2^{\text {nd }}$ period fire control problem), which depend on the outcome of the $1^{\text {st }}$ MCDM sub-problem. Furthermore, any feasible fire assignment plan is a combination of two-period fire assignment strategies, and each fire assignment strategy consists of multiple actions that how many ammunitions of a weapon are assigned to a target. Because there exist joint damages between a pair of ammunitions, action-interdependences need to be noticed. Therefore, any fire assignment plan is just a system similar to the plan in an SDM problem. At length, prioritizations and cooperations among targets are worth being paid attentions to, which results in the overall evaluation of a fire assignment strategy cannot be a linear aggregation of the evaluations of the fire assignment with respect to respective targets, just like the formulations of $F_{1}$ and $F_{2}$ aforementioned. In fact, both prioritizations and cooperations are criteria-interdependences in the SDM. Therefore, we can come a conclude, from the above solution process of the fire control problem, some complex decision making problems can be well solved under the framework of the SDM, which also illustrate that the SDM is meaningful and worth being developed. 


\section{Conclusions}

In this paper, a kind of complex multi-criteria decision making (MCDM) problems, which cannot be well solved by existing MCDM methods, have been analyzed in detail in terms of criteria, alternatives, decision making environment and time sequential connections etc., and then a systematic decision making (SDM) model, in which each plan is a system and criteria constitute a criteria system, has been developed in order to deal with such MCDM problems.

As mentioned in Section 1, MCDM problems are essentially a special class of multiobjective programming problems, and we try to solve them by tackling the corresponding multi-objective programming models. It seems meaningless to develop various MCDM models rather than devise some proper multi-objective programming methods. In fact, the significances of MCDM models should not be underestimated. Lots of multi-objective programming problems are hard to be solved, whereas MCDM models can reveal intrinsic characteristics of a typical class of multi-objective programming problems, based on which this class of problems are easily transformed into corresponding single-objective programming ones and then be solved accurately and simply, just as the relation between Expressions (1) and (3). As an improvement and supplement of existing MCDM models, the SDM model in this paper tries to handle more multi-objective programming problems which can not be well dealt with by traditional MCDM models. Some complex multiobjective programming problems concerned, which consume our a lot of time and energies and even are impossible to be solved by means of multi-objective programming models and methods, can be well analyzed by the SDM model so as to find out feasible methods to handle them. At least, the SDM model, which provides another alternative idea to cope with some complex multi-objective programming problems, can make it more probably to solve these problems successfully. Thus, the SDM is meaningful.

However, the approach of each step in the SDM model depends on the concrete problem, thus we must be clear on the specialties of the problem besides its common characteristics of such a kind of complex MCDM problems. Therefore, it is meaningful to apply the SDM model into some actual problems so as to develop SDM methods. Due to limitation of spaces, it is hard to design some practical approaches in detail, so the innovation of methods is restricted. In our future work, we will try to handle some practical complex MCDM problems in various fields under the framework of the SDM model, contribute ourselves to perfect the model, develop a serial of SDM methods to quantitatively analyze intrinsic characteristics of the problems, and finally solve them.

\section{Acknowledgements}

The work on this paper was supported by the National Natural Science Foundation of China (No. 71501186). 


\section{References}

Amin, G. R.; Sadeghi, H. 2010. Application of prioritized aggregation operators in preference voting, International Journal of Intelligent Systems 25(10): 1027-1034. https://doi.org/10.1002/int.20437

Angilella, S.; Greco, S.; Lamantia, F.; Matarazzo, B. 2004. Assessing non-additive utility for multicriteria decision aid, European Journal of Operational Research 158(3): 734-744. https://doi.org/10.1016/S0377-2217(03)00388-6

Antuchevičiene, J.; Zavadskas, E. K.; Zakarevičius, A. 2010. Multiple criteria construction management decisions considering relations between criteria, Technological and Economic Development of Economy 16(1): 109-125. https://doi.org/10.3846/tede.2010.07

Bellman, R. E.; Zadeh, L. A. 1970. Decision-making in a fuzzy environment, Management Science 17(4): 141-164. https://doi.org/10.1287/mnsc.17.4.B141

Calizaya, A.; Meixner, O.; Bengtsson, L.; Berndtsson, R. 2010. Multi-criteria decision analysis (MCDA) for integrated water resources management (IWRM) in the Lake Poopo basin, Bolivia, Water Resources Management 24(10): 2267-2289. https://doi.org/10.1007/s11269-009-9551-x

Campanella, G.; Ribeiro, R. A. 2011. A framework for dynamic multiple-criteria decision making, Decision Support Systems 52(1): 52-60. https://doi.org/10.1016/j.dss.2011.05.003

Carlsson, C.; Fullèr, R. 1995. Multiple criteria decision making: the case for interdependence, Computers Operational Research 22(3): 251-260. https://doi.org/10.1016/0305-0548(94)E0023-Z

Chen, L. H.; Xu, Z. S. 2015. A new prioritized multi-criteria outranking method: the prioritized PROMETHEE, Journal of Intelligent \& Fuzzy Systems 29(5): 2099-2110. https://doi.org/10.3233/IFS-151686

da Costa Pereira, C.; Dragoni, M.; Pasi, G. 2011. Multidimensional relevance: prioritized aggregation in a personalized Information Retrieval setting, Information Processing and Management 48(2): 340-357. https://doi.org/10.1016/j.ipm.2011.07.001

Dey, B.; Bairagi, B.; Sarkar, B.; Sanyal, S. K. 2016. Multi objective performance analysis: a novel multicriteria decision making approach for a supply chain, Computers \& Industrial Engineering 94: 105124. https://doi.org/10.1016/j.cie.2016.01.019

Dweiri, F.; Kumar, S.; Khan, S. A.; Jain, V. 2016. Designing an integrated AHP based decision support system for supplier selection in automotive industry, Expert Systems with Applications 62: 273-283. https://doi.org/10.1016/j.eswa.2016.06.030

Fan, Z. P.; Feng, B. 2009. A multiple attributes decision making method using individual and collaborative attribute data in a fuzzy environment, Information Sciences 179(20): 3603-3618. https://doi.org/10.1016/j.ins.2009.06.037

Ghosh, S.; Chakraborty, T.; Saha, S.; Majumder, M.; Pal, M. 2016. Development of the location suitability index for wave energy production by ANN and MCDM techniques, Renewable and Sustainable Energy Reviews 59: 1017-1028. https://doi.org/10.1016/j.rser.2015.12.275

Grabisch, M.; Marichal, J. L.; Mesiar, R.; Pap, E. 2009. Aggregation functions. Cambridge: Cambridge University Press. https://doi.org/10.1017/CBO9781139644150

Huang, W. B.; Wu, Z. Q.; Chen, Y. H. 2010. Prioritized attributes based threat evaluation model for the aerial targets, Ship Science and Technology 32(9): 59-62.

Kaliszewski, I.; Miroforidis, J.; Podkopaev, D. 2012. Interactive multiple criteria decision making based on preference driven evolutionary multiobjective optimization with controllable accuracy, European Journal of Operational Research 216(1): 188-199. https://doi.org/10.1016/j.ejor.2011.07.013

Koele, P. 1995. Multiple attribute decision making: an introduction. Thousand Oaks: Sage Publications.

Kornbluth, J. S. H. 1992. Dynamic multi-criteria decision making, Journal of Multi-Criteria Decision Analysis 1(2): 81-92. https://doi.org/10.1002/mcda.4020010204 
Kumar, A.; Pandey, A. C. 2016. Geoinformatics based groundwater potential assessment in hard rock terrain of Ranchi urban environment, Jharkhand state (India) using MCDM-AHP techniques, Groundwater for Sustainable Development 2-3: 27-41. https://doi.org/10.1016/j.gsd.2016.05.001

Liao, Z. 1998. A systematic integration model to support complex decision-making in a dynamic environment, Systems Research and Behavioral Science 15(1): 33-45. https://doi.org/10.1002/(SICI)1099-1743(199801/02)15:1<33::AID-SRES189>3.0.CO;2-6

Malekmohammadi, B.; Rahimi Blouchi, L. 2014. Ecological risk assessment of wetland ecosystems using Multi Criteria Decision Making and Geographic Information System, Ecological Indicators 41: 133-144. https://doi.org/10.1016/j.ecolind.2014.01.038

Murofushi, T.; Sugeno, M. 1989. An interpretation of fuzzy measure and the Choquet integral as an integral with respect to a fuzzy measure, Fuzzy Sets and Systems 29(2): 201-227. https://doi.org/10.1016/0165-0114(89)90194-2

Rajabi, S.; Kilgour, D. M.; Hipel, K. W. 1998. Modeling action-interdependence in multiple criteria decision making, European Journal of Operational Research 110(3): 480-508. https://doi.org/10.1016/S0377-2217(97)00318-4

Sugeno, M. 1974. Theory of fuzzy integrals and its applications. Tokyo: Tokyo Institute of Technology.

Townsend, J. T.; Busemeyer, J. 1995. Dynamic representation of decision-making, in R. Port, T. V. Gelder (Eds.). Mind in motion: dynamics, behavior and cognition 1995. MIT Press.

Vahdat, K.; Smith, N. J.; Amiri, G. G. 2014. Fuzzy multicriteria for developing a risk management system in seismically prone areas, Socio-Economic Planning Sciences 48(4): 235-248. https://doi.org/10.1016/j.seps.2014.05.002

Verma, R.; Sharma, B. 2016. Prioritized information fusion method for triangular fuzzy information and its application to multiple attribute decision making, International Journal of Uncertainty Fuzziness and Knowledge-Based Systems 24(2): 265-289. https://doi.org/10.1142/S0218488516500136

Wang, J.; Yang, Y.; Sui, J.; Jin, H. 2016. Multi-objective energy planning for regional natural gas distributed energy: a case study, Journal of Natural Gas Science and Engineering 28: 418-433. https://doi.org/10.1016/j.jngse.2015.12.008

Wang, J. J.; Jing, Y. Y.; Zhang, C. F.; Zhao, J. H. 2009. Review on multi-criteria decision analysis aid in sustainable energy decision-making, Renewable and Sustainable Energy Reviews 13(9): 2263-2278. https://doi.org/10.1016/j.rser.2009.06.021

Weng, S. Q.; Huang, G. H.; Li, Y. P. 2010. An integrated scenario-based multi-criteria decision support system for water resources management and planning-a case study in the Haihe River Basin, Expert Systems with Applications 37(12): 8242-8254. https://doi.org/10.1016/j.eswa.2010.05.061

Xu Y. J.; Li, D. F.; Zhang, N.; Wu, Z. Q. 2010. Target type recognition method based on intuitionistic fuzzy set theory and POWA operators, Electronics Optics \& Control 17(11): 22-25.

$\mathrm{Xu}, \mathrm{Z}$. S. 2010. Choquet integrals of weighted intuitionistic fuzzy information, Information Sciences 180(5): 726-736. https://doi.org/10.1016/j.ins.2009.11.011

Yager, R. R. 2004. Modeling prioritized multicriteria decision making, IEEE Transactions on Systems, Man, and Cybernetics, Part B: Cybernetics 34(6): 2396-2404. https://doi.org/10.1109/TSMCB.2004.837348

Yager, R. R. 2008. Prioritized aggregation operators, International Journal of Approximate reasoning 48(1): 263-274. https://doi.org/10.1016/j.ijar.2007.08.009

Yager, R. R.; Rybalov, A. 1998. Full reinforcement operators in aggregation techniques, IEEE Transactions on Systems, Man, and Cybernetics, Part B: Cybernetics 28(6): 757-769. https://doi.org/10.1109/3477.735386 
Yan, H. B.; Huynh, V. N.; Nakamori, Y.; Murai, T. 2011. On prioritized weighted aggregation in multicriteria decision making, Expert Systems with Applications 38(1): 812-823. https://doi.org/10.1016/j.eswa.2010.07.039

Yu, X. H.; Xu, Z. S. 2012. Graph-based multi-agent decision making, International Journal of Approximate Reasoning 53(4): 502-512. https://doi.org/10.1016/j.ijar.2011.12.002

Yu, X. H.; Xu, Z. S. 2013. Prioritized intuitionistic fuzzy aggregation operators, Information Fusion 14(1): 108-116. https://doi.org/10.1016/j.inffus.2012.01.011

Xiaohan YU. Doctor, Lecturer. College of Command Information Systems, PLA University of Science and Technology. Doctor of military operational research, PLA University of Science and Technology (2014). Author of more than 20 scientific articles. Research interests: multi-criteria decision making, data mining, and military operational research.

Zeshui XU. Doctor, Professor. College of Sciences, PLA University of Science and Technology. Doctor of management science and engineering, Southeast University (2003). Distinguished Young Scholar of the National Natural Science Foundation of China. The Chang Jiang Scholars of the Ministry of Education of China. Author of more than 450 scientific articles and 7 books. Research interests: information fusion, decision making, and fuzzy sets.

Junquan HU. Master, Associate Professor. College of Communications Engineering, PLA University of Science and Technology. First degree in engineering, PLA Institute of Communications Engineering (1996). Master of engineering (1999). Research interests: wireless communication, wireless signal processing.

Shousheng LIU. Doctor, Professor. College of Sciences, PLA University of Science and Technology. Master of mathematics, Beijing Normal Universion (1994). Doctor of signal processing, Nanjing University of Aeronautics and Astronautics (2005). Author of more than 50 scientific articles. Research interests: decision making, information processing, and stochastic processes. 